\title{
ESTUDIO DE MONOCAPAS DE BROMUROS ALQUÍLICOS EN SUPERFICIES HIDROGENADAS DE SILICIO (100)
}

\author{
Yris Del Pilar Obregón Rodríguez ${ }^{* a}$, César R. Mayor Sánchez ${ }^{\mathrm{b}}$
}

\begin{abstract}
RESUMEN
Las monocapas orgánicas de cadenas alquílicas saturadas enlazadas al sustrato de silicio son usadas para el desarrollo de dispositivos con importantes propiedades electrónicas.

En este estudio, gracias al empleo de las moléculas 10-bromo-1-deceno y 11-bromo1-undeceno en superficies de silicio, se logra la formación de un sistema potencialmente aplicable en la modificación de los grupos terminales de la cadena carbonada en las monocapas orgánicas, es decir la funcionalización de la monocapa permitiendo un mejor transporte de carga a través de las moléculas.

La superficie de silicio (libre de óxidos) se pasivó por hidrogenación. Respecto a la formación de la monocapa orgánica, la superficie de silicio hidrogenada fue sometida a $200^{\circ} \mathrm{C}$ con cada una de las moléculas orgánicas mencionadas.

Tanto la superficie hidrogenada como las monocapas orgánicas fueron caracterizadas por medición del ángulo de contacto entre gotas de agua y la superficie, y microscopía de fuerza atómica.

Finalmente, mediante cálculos moleculares a nivel 3-21(G) sobre clusters de Si7H6, $\mathrm{Si}_{7} \mathrm{H}_{15} \mathrm{C}_{10} \mathrm{H}_{20} \mathrm{Br}$ y $\mathrm{Si}_{7} \mathrm{H}_{15} \mathrm{C}_{11} \mathrm{H}_{22} \mathrm{Br}$, se proponen posibles reacciones de formación de las monocapas orgánicas estudiadas experimentalmente.
\end{abstract}

Palabras clave: monocapas alquílicas en $\mathrm{Si}$ (100), ángulo de contacto, microscopía de fuerza atómica (AFM), clusters de hidruros de silicio.

\section{STUDY OF ALKYL-BROMIDE MONOLAYERS ON HYDROGEN- TERMINATED SILICON (100) SURFACES}

\begin{abstract}
The organic monolayers of saturated alkyl chains linked to silicon substrates are used for the development of devices with important electronic properties. In this study, thanks to the use of 10-bromine-1-decene and 11-bromine-1-undecene molecules on silicon surfaces, the formation of a system potentially applicable in the modification of the end groups of the carbon chain is achieved in the organic monolayers, that is to say the functionalization of the monolayer allowing a better charge transport through the molecules.
\end{abstract}

\footnotetext{
${ }^{a}$ Facultad de Ciencias e Ingeniería - Sección Química, Pontificia Universidad Católica del Perú (PUCP), Av. Universitaria 1801, San Miguel, Lima 32, Perú, yares2605@gmail.com

${ }^{\mathrm{b}}$ Facultad de Ingeniería Industrial - Mecánica (FIME), Universidad Tecnológica del Perú (UTP).
} 
The silicon surface (oxide-free) was passivated by hydrogenation. Respect to the formation of the organic monolayer, the hydrogen-terminated silicon surface was subjected to $200^{\circ} \mathrm{C}$ with each of the mentioned organic molecules.

Both the hydrogenated surface and the organic monolayers were characterized by the measurement of the contact angle between water droplets and the surface, and atomic force microscopy.

Finally, by means of molecular calculations at levels 3-21(G) on clusters of Si7H6, $\mathrm{Si}_{7} \mathrm{H}_{15} \mathrm{C}_{10} \mathrm{H}_{20} \mathrm{Br}$ and $\mathrm{Si}_{7} \mathrm{H}_{15} \mathrm{C}_{11} \mathrm{H}_{22} \mathrm{Br}$, possible reactions of formation of the organic monolayers previously studied experimentally, are proposed.

Key words: Alkyl monolayers on Si (100), contact angle, atomic force microscopy (AFM), silicon hydride clusters.

\section{INTRODUCCIÓN}

Los trabajos sobre las superficies de silicio han sido profundamente estudiados en los últimos años debido a su importancia en la tecnología moderna. Las propiedades electrónicas de moléculas orgánicas depositadas sobre los sustratos de silicio proporcionan un gran estímulo para investigar y diseñarlos como componentes electrónicos ${ }^{1,2}$.

Asimismo, cabe comentar que, la evolución de la tecnología electrónica ha estado ligada a la continua miniaturización de componentes y multiplicación de su velocidad de proceso computacional, dado por la popular Ley de Moore ${ }^{3,4}$.

La investigación en el desarrollo, entre el campo de la electrónica y la química orgánica, se basa en la aplicación práctica de los materiales orgánicos en dispositivos optoelectrónicos debido a su capacidad de graduar bandas electrónicas en el semiconductor ${ }^{5}$.

La facilidad de fabricación y la posibilidad de producir estructuras en capas controladas con precisión hacen de este material adecuado para su uso en una amplia gama de campos, tales como óptica, microelectrónica, optoelectrónica, detección química o aplicaciones biomédicas ${ }^{3}$.

Las metodologías sintéticas brindadas para la fabricación de estos dispositivos modulan las propiedades de este tipo de materiales, potenciando su aplicabilidad. Las características peculiares generadas por estos materiales, unidos a su mayor disponibilidad y accesibilidad, ligereza o facilidad de procesamiento, hacen que los materiales orgánicos puedan ser fabricados reduciendo la cantidad de material empleado. Es por ello, que la búsqueda de nuevos materiales semiconductores orgánicos es motivo de muchas investigaciones actualmente. La amplia variedad de semiconductores orgánicos los hay tanto de naturaleza molecular como polimérica (polímeros conductores). Sin embargo, los derivados moleculares pueden ser sintetizados de forma reproducible y con una estructura perfectamente conocida, hecho que refuerza su potencial en su aplicación tecnológica ${ }^{3,4}$. 
Las aplicaciones tecnológicas de estos materiales encuentran su uso práctico en biosensores, transistores de efecto campo MOSFET (metal-oxide semiconductor field effect transistor), diodos orgánicos, dispositivos emisores de luz, células solares orgánicas OSCs (Organic Solar Cells), dispositivos de almacenamiento de memoria, filtrados fotodetectores, sensores ópticos, entre otros ${ }^{3,4}$.

La ventaja en la fabricación de estos semiconductores unidos covalentemente a las moléculas orgánicas radica en la funcionalización bajo condiciones atmosféricas, dando como resultado las monocapas orgánicas en superficies de silicio (sin la capa interfacial del óxido de silicio), lo cual son una interesante nueva clase de monocapas en sustratos de sólidos que es un tópico de intensa investigación.

Debido a que el enlace covalente $\mathrm{Si}-\mathrm{C}$, que es formado entre la superficie de silicio y el compuesto orgánico, las monocapas muestran una notable estabilidad tanto térmicamente como químicamente ${ }^{6,7}$.

En este estudio se recurre a la reacción entre bromuros alquílicos insaturados y la superficie hidrogenada de Si (100) para formar monocapas orgánicas ordenadas sobre sustratos de silicio, lo cual ofrece la posibilidad de desarrollar dispositivos con importantes propiedades electrónicas. Tanto la superficie hidrogenada (realizada por la reacción de pasivación ${ }^{8,9}$ ) como las monocapas orgánicas son caracterizadas por medición del ángulo de contacto entre gotas de agua y la superficie en cuestión, como la microscopía de fuerza atómica (AFM) y también mediante cálculos teóricos GaussView 5.0 y Gaussian $09 \mathrm{~W}^{10}$.

\section{PARTE EXPERIMENTAL}

\section{Limpieza previa y pasivación del sustrato de silicio}

Se tomaron obleas de silicio de área aproximada de $1 \mathrm{x} 1 \mathrm{~cm}^{2}$, las cuales fueron lavadas y desengrasadas sucesivamente con etanol y acetona, y luego secadas con $\mathrm{N}_{2}$. Después se lavaron con solución de $\mathrm{H}_{2} \mathrm{O}_{2}$ al $30 \% \mathrm{y} \mathrm{H}_{2} \mathrm{SO}_{4}$ (1:2) comúnmente conocida como solución piraña por 2 horas a $90^{\circ} \mathrm{C}$. Posteriormente, las piezas de silicio fueron enjuagadas con agua destilada por cinco minutos.

Luego fueron tratadas pasivando las piezas de silicio de $1 \mathrm{x} 1 \mathrm{~cm}^{2}$ con una solución de HF al $2 \%$ por espacio de dos minutos, con la finalidad de obtener grupos funcionales $\mathrm{Si}-\mathrm{H}$ en la superficie.

\section{Preparación de monocapas de bromuros alquílicos}

Las monocapas de bromuros alquílicos, tanto de 10-bromo-1-deceno como de 11-bromo-1undeceno, fueron preparadas por inmersión de las obleas en un tubo de ensayo con $2 \mathrm{ml}$ del respectivo precursor previamente desoxigenado haciendo burbujear nitrógeno por toda la noche y se colocó en un baño de aceite aproximado a $200^{\circ} \mathrm{C}$ por 20 horas bajo flujo de $\mathrm{N}_{2}$. Luego de la formación de la monocapa se retira del baño y finalmente se secó con gas $\mathrm{N}_{2}$. 


\section{Caracterización de monocapas de bromuros alquílicos}

Ángulo de contacto. Empleando $0,3 \mathrm{ml}$ de agua destilada sobre la superficie preparada, se midió el ángulo de contacto respecto al sustrato. Tales mediciones se realizaron tanto en el Laboratorio 2 de Química (PUCP) como en el Laboratorio de Materiales Nanoestructurados (UNI). En este último caso, se empleó MATLAB 7.0 para el cálculo del ángulo a partir de tratamiento de imágenes fotografiadas de las gotas.

Microscopía de fuerza atómica (AFM). El registro de las imágenes por AFM de las muestras de silicio modificadas con bromuros alquílicos, fue obtenido con el equipo EasyScan 2 del fabricante Nanosurf ${ }^{\circledR}$, en modo de contacto con resolución de 256 x 256 píxel, sistema de piezo barrido de $5 \times 5 \mathrm{~mm}^{2}$ y sonda tipo ultralever de $600 \mathrm{~nm}$ de diámetro. Estos ensayos fueron realizados en el Laboratorio de Fuerza Atómica del Instituto Peruano de Energía Nuclear (IPEN) siendo las mejores imágenes obtenidas usando el equipo mencionado.

Cálculos energéticos empleando clusters de silicio. Empleando clusters de $\mathrm{Si}_{7} \mathrm{H}_{16}$,

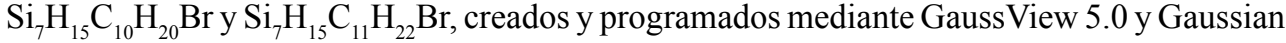
09W, se calculó el cambio de energía en la reacción:

$\mathrm{Si}_{7} \mathrm{H}_{16}+\mathrm{C}_{\mathrm{n}} \mathrm{H}_{2 \mathrm{n}-1} \mathrm{Br} \rightarrow \mathrm{Si}_{7} \mathrm{H}_{15} \mathrm{C}_{\mathrm{n}} \mathrm{H}_{2 \mathrm{n}} \mathrm{Br}(\mathrm{n}=10,11)$.

Los cálculos se realizaron a nivel 3-21(G).

\section{RESULTADOS Y DISCUSIÓN}

\section{Ángulo de contacto}

Las micrografías para gotas de agua sobre el sustrato de silicio muestran que la superficie no pasivada forma un ángulo de contacto de $52,1^{\circ}$, debido a la presencia de fuerzas intermoleculares (ligero carácter hidrofóbico) en la superficie (figura 1).

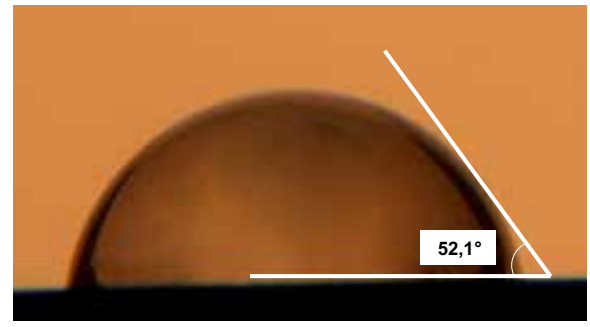

Figura 1. Ángulo de contacto de una superficie no pasivada $\left(\mathrm{SiO}_{2}\right)$. 
En cambio, para las monocapas orgánicas sobre silicio, se observa un comportamiento altamente hidrofóbico con ángulos de contacto de $71,7^{\circ}$ y $75,4^{\circ}$ de las moléculas orgánicas silicio-decano-bromo $\mathrm{Si}-\mathrm{C}_{10} \mathrm{H}_{20}-\mathrm{Br}$ y silicio-undecano-bromo $\mathrm{Si}-\mathrm{C}_{11} \mathrm{H}_{22}-\mathrm{Br}$, respectivamente (figuras 2 y 3 ). Es decir, indicando una mayor hidrofobicidad entre el agua y la cadena alquílica auto-ensamblada sobre la superficie de silicio.

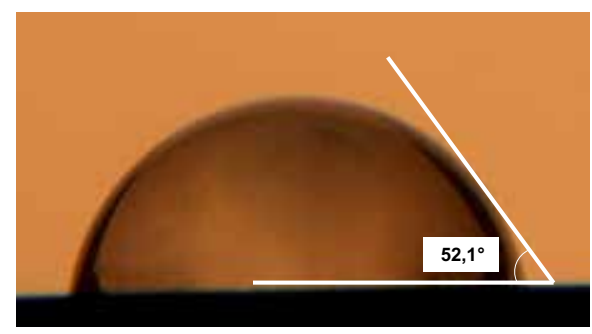

Figura 2. Ángulo de contacto de una monocapa orgánica sobre silicio (silicio-decano-bromo $\mathrm{Si}-\mathrm{C}_{10} \mathrm{H}_{20}-\mathrm{Br}$ ).

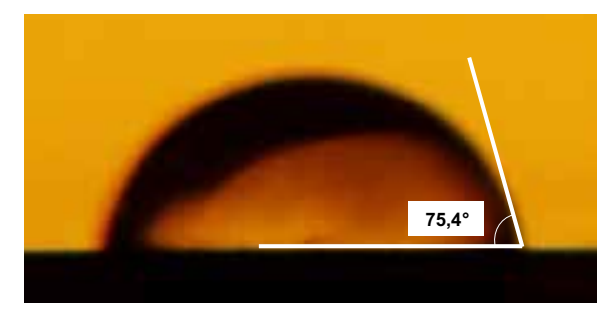

Figura 3. Ángulo de contacto de una monocapa orgánica sobre silicio (silicio-undecano-bromo $\mathrm{Si}_{-} \mathrm{C}_{11} \mathrm{H}_{22}-\mathrm{Br}$ ).

\section{Microscopía de fuerza atómica (AFM)}

El análisis AFM en la superficie de silicio sin tratamiento (figura 4) revela una muestra con una textura superficial casi lisa, notándose una superficie homogénea con casi la misma tonalidad en todo el sustrato. Lo cual conlleva a deducir la presencia del óxido nativo del silicio ( $\mathrm{SiO} 2)$ como también podría ser de otras sustancias producto de la exposición al medio ambiente. 


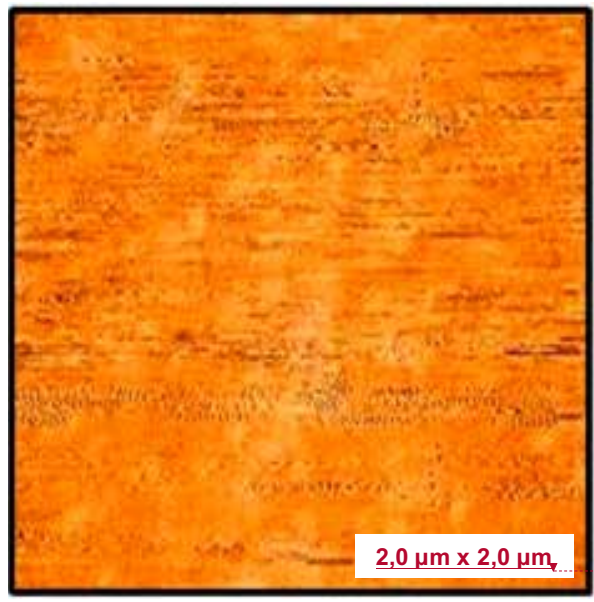

Figura 4. Superficie no tratada.

El resultado del análisis AFM en la superficie de silicio bajo el tratamiento de pasivación por hidrogenación (figura 5) presenta una amplia gama de texturas superficiales que indican que el ataque del HF en casi todo el sustrato fue óptimo, lo cual nos sugiere cambios en el perfil topográfico del sustrato de silicio pasivado, mostrando una imagen rugosa y con zonas granuladas de color blanco y de tamaño irregular en casi toda la superficie. También se logra observar, en pequeñas partes del sustrato, manchas de color marrón oscuras deduciendo que corresponden a zonas con poco ataque o zonas defectuosas del material que no fueron afectadas por el tratamiento de pasivación.

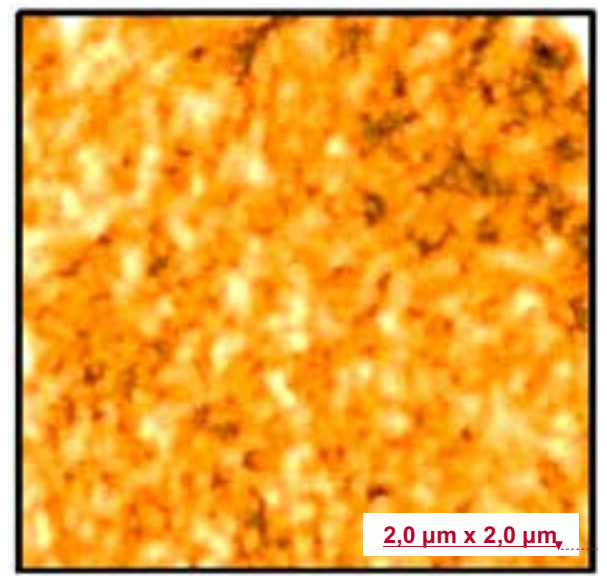

Figura 5. Superficie hidrogenada $(\mathrm{Si}-\mathrm{H})$. 
En cuanto a la monocapa orgánica auto-ensamblada en el sustrato de silicio (figuras 6 y 7: silicio-decano-bromo y silicio-undecano-bromo, respectivamente), también observamos diferentes tonalidades, lo cual nos sugiere nuevamente cambios en el perfil morfológico de ambas muestras mencionadas.

La imagen topográfica de la figura 6 presenta zonas nebulosas, formando geometrías rectangulares en casi toda la superficie; a diferencia de la figura 7 que muestra zonas granuladas muy tupidas de color blanco por todo el sustrato. Lo cual indicaría que en ambas muestras (figuras 6 y 7) se produjo efectivamente la respectiva formación de las monocapas orgánicas en las superficies de silicio.

Cabe resaltar que el AFM es una técnica de caracterización topográfica de superficies, no proporcionando información sobre el tipo de molécula presente en el sustrato, tan sólo presumir la presencia de moléculas empleadas en cada uno de los tratamientos.

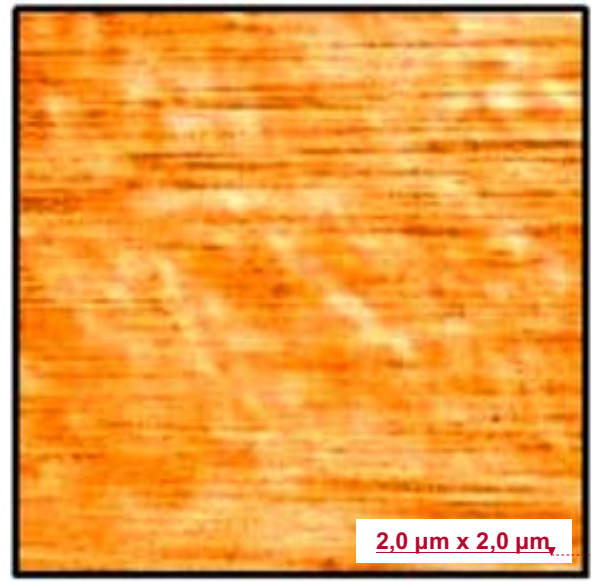

Figura 6. Superficie auto-ensamblada de silicio-decano-bromo $\left(\mathrm{Si}_{10} \mathrm{C}_{10} \mathrm{H}_{20}-\mathrm{Br}\right)$.

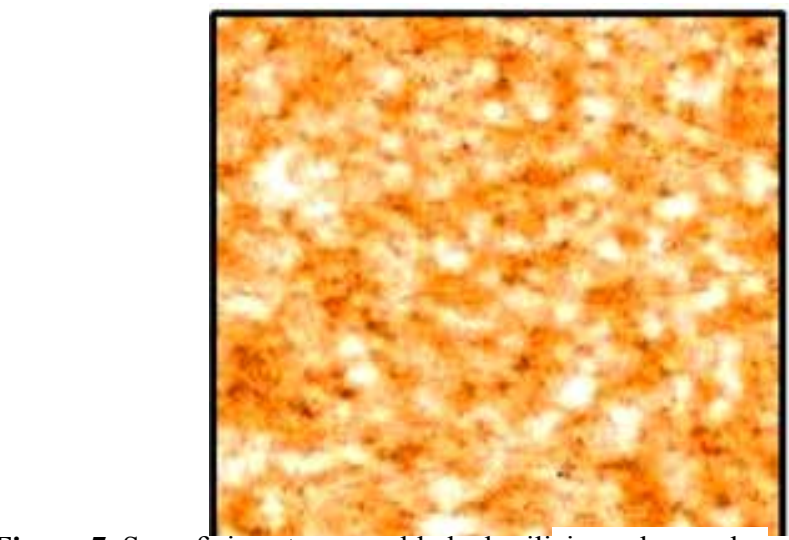

Figura 7. Superficie auto-ensamblada de silicio-undecano-bromo $\left(\mathrm{Si}-\mathrm{C}_{11} \mathrm{H}_{22}-\mathrm{Br}\right)$. 


\section{Cálculos energéticos empleando clusters de silicio}

Para la adsorción de ambos precursores orgánicos se usó valores obtenidos descritos en la tabla 1, determinándose el valor energético en cada reacción (tabla 2).

Con esta variación de bajo valor de energía obtenido del modelo propuesto, podemos predecir la reacción para la formación de monocapas de bromuros alquílicos sobre hidruros de silicio, generando también, con este valor, que la cadena orgánica tienda a ser repelida por la superficie de silicio (figura 8).

Tabla 1. Valores energéticos de los clusters en unidades de Hartree.

\begin{tabular}{|c|c|}
\hline Clusters & Energía total (Hartree) \\
\hline $\mathrm{Si}_{7} \mathrm{H}_{6}$ & $-2021,03$ \\
\hline $\mathrm{C}_{10} \mathrm{H}_{19} \mathrm{Br}$ & $-2948,16$ \\
\hline $\mathrm{Si}_{7} \mathrm{H}_{15} \mathrm{C}_{10} \mathrm{H}_{20} \mathrm{Br}$ & $-4968,68$ \\
\hline $\mathrm{C}_{11} \mathrm{H}_{21} \mathrm{Br}$ & $-2986,98$ \\
\hline $\mathrm{Si}_{7} \mathrm{H}_{15} \mathrm{C}_{11} \mathrm{H}_{22} \mathrm{Br}$ & $-5007,52$ \\
\hline
\end{tabular}

Tabla 2. Variación de la energía $(\mathrm{kJ} / \mathrm{mol})$ de cada reacción química.

\begin{tabular}{|c|c|}
\hline Reacción química & Variación de la energía (kJ/mol) \\
\hline $\mathrm{Si}_{7} \mathrm{H}_{16}+\mathrm{C}_{10} \mathrm{H}_{19} \mathrm{Br} \rightarrow \mathrm{Si}_{7} \mathrm{H}_{15} \mathrm{C}_{10} \mathrm{H}_{20} \mathrm{Br}$ & $-1559,55$ \\
\hline $\mathrm{Si}_{7} \mathrm{H}_{16}+\mathrm{C}_{11} \mathrm{H}_{21} \mathrm{Br} \rightarrow \mathrm{Si}_{7} \mathrm{H}_{15} \mathrm{C}_{11} \mathrm{H}_{22} \mathrm{Br}$ & $-1612,06$ \\
\hline
\end{tabular}

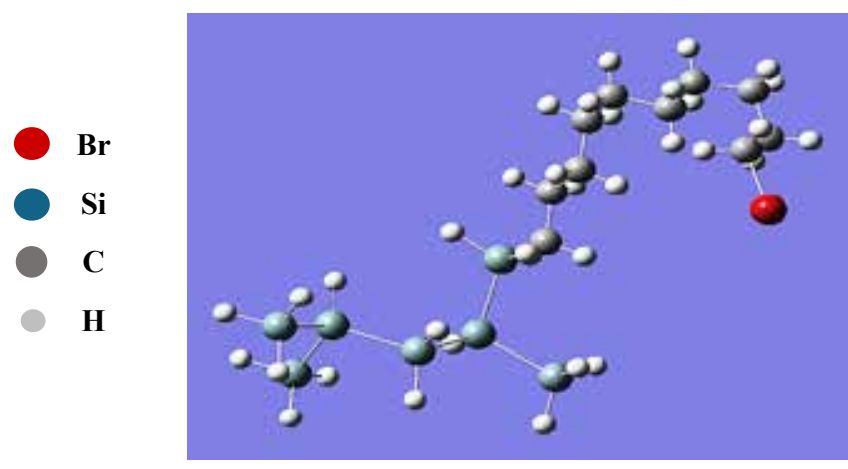

Figura 8. Superficie auto-ensamblada de la molécula orgánica. 


\section{CONCLUSIONES}

- Las monocapas de bromuros alquílicos, en la superficie de silicio, tienden a formar un empaquetamiento ordenado, que resulta de una adsorción energéticamente favorable, y con alto grado de repulsión entre la cadena orgánica y el sustrato de silicio.

- Se ha verificado que gracias a la medida del ángulo de contacto y AFM, se logró comprobar la presencia de monocapas alquílicas de Br-terminadas con una activación térmica aproximada a $200^{\circ} \mathrm{C}$ y en condiciones de protección atmosférica menos exigentes que las reportadas por Cohen y colaboradores.

- Se pudieron predecir con la energía obtenida mediante cálculos moleculares a nivel 3-21(G) sobre clusters de $\mathrm{Si}_{7} \mathrm{H}_{16}, \mathrm{Si}_{7} \mathrm{H}_{15} \mathrm{C}_{10} \mathrm{H}_{20} \mathrm{Br} \mathrm{y} \mathrm{Si}_{7} \mathrm{H}_{15} \mathrm{C}_{11} \mathrm{H}_{22} \mathrm{Br}$, una reacción favorable a la formación de monocapas orgánicas de cadenas de $\mathrm{Br}$-alquílicas sobre la superficie de silicio.

\section{AGRADECIMIENTOS}

Los autores agradecen el apoyo brindado para este trabajo de investigación al Dr. Maynard Kong de la Sección Química y Dirección de Gestión a la Investigación (DGI) de la PUCP. Asimismo, al doctor Abel Gutarra del Laboratorio de Materiales Nanoestructurados de la Facultad de Ciencias (UNI), como también, al doctor José Solís, del Laboratorio de Microscopía de Fuerza Atómica - Instituto Peruano de Energía Nuclear (IPEN).

\section{REFRERENCIAS BIBLIOGRÁFICAS}

1. Y. S. Cohen YS, A. Vilan A, I. Ron I, D. Cahen D. Hydrolysis Improves Packing Density of Bromine-Terminated Alkyl-Chain, Silicon-Carbon Monolayers Linked to Silicon J Phys Chem C. 2009; 113(15): 6174-6181.

2. Midwood KS, Carolus MD, Danahy MP, Schwarzbauer JE, Schwartz J. Easy and efficient bonding of biomolecules to an oxide surface of silicon. Langmuir. 2004; 20: 5501-5505.

3. Torres-Costa V, Martín-Palma RJJ. Application of nanostructured porous silicon in the field of optics. A review. J Mater Sci. 2010; 45(11): 2823-2838.

4. Más Montoya M. Síntesis de Nuevos Sistemas Heteroacénicos y Estudio de sus Propiedades como Semiconductores Orgánicos para su Aplicación en Electrónica Molecular. [Tesis doctoral]. Murcia: Universidad de Murcia; 2015.

5. Honeyman B, Bedzyk M, Hersam M, Hua Jin C, Kinser R. Stability Study of SelfAssembled Monolayers on Silicon(111). Nanoscape. Spring 2005; 2(1): 89-95.

6. Sieval AB, Demirel AL, Nissink JWM, Linford MR, Van der Maas JH, De Jeu WH, et al. Highly Stable $\mathrm{Si}-\mathrm{C}$ Linked Functionalized Monolayers on the Silicon (100) Surface. Langmuir. 1998; 14 (7): 1759-1768.

7. Sieval AB, Huisman CL, Schönecker A, Schulman's FM, Van Der Heide ASH, Goossens A, et. al. Silicon Surface Passivation by Organic Monolayers: Minority Charge Carrier 
Lifetme Measurements and Kelvin Probe Investigations. J Phys Chem B. 2003; 107: 6846-6852.

8. Sieval AB, Opitz R, Maas HPA, Schoeman MG, Meijer G, Vergeldts FJ, et al. Monolayers of 1-Alkynes on the H-Terminated Si(100) Surface. Langmuir. 2000; 16(26): 1035910368.

9. Sieval AB, Vleeming V, Zuilhof H, Sudhölter EJR. An Improved Method for the Preparation of Organic Monolayers of 1-Alkenes on Hydrogen-Terminated Silicon Surfaces. Langmuir. 1999; 15: 8288-8291.

10. Sieval AB, Linke R, Heij G, Meijer G, Zuilhof H, Sudhölter EJR. Amino-Terminated Organic Monolayers on Hydrogen-Terminated Silicon Surfaces. Langmuir. 2001; 17(24): 7554-7559. 